\title{
The distribution of plaques in the cerebrum in multiple sclerosis
}

\author{
BETTY BROWNELL AND J. TREVOR HUGHES \\ From the Departments of Neurology and Pathology, Radcliffe Infirmary, Oxford
}

One of the fundamental problems posed by multiple sclerosis is concerned with the distribution of the pathological lesions called plaques. The literature concerning the histopathology of this disease is immense but a recent review by Lumsden (1955) did not refer to any paper in which the position of plaques in the cerebrum had been determined with any exactitude although such an exact study was made on the spinal cord of eight cases by Fog (1950), who found that spinal plaques occurred consistently in certain regions. This work was based on histological sections cut transversely through the spinal cord, a technique also applicable to the brain-stem but not essential in the cerebrum where the position of plaques can be seen sufficiently well by the naked eye.

The present study was undertaken to determine with some precision the position and relative frequency of plaques seen macroscopically in the cerebral hemispheres in a series of necropsied cases of multiple sclerosis.

\section{METHOD}

Twenty-two unselected cases were studied, comprising every case of multiple sclerosis coming to necropsy at the Radcliffe Infirmary during the four years 1958 to 1961 .

The fixed cerebral hemispheres were cut coronally at $1 \mathrm{~cm}$. intervals, the first cut being made immediately in front of the mammillary bodies. This procedure is the usual practice in the Department of Pathology, and the resulting coronal slices anterior to the first cut are called $\mathrm{A} 1,2,3$, etc. and those posterior $\mathrm{P1}, 2,3$, etc. The inspection of these $1 \mathrm{~cm}$. coronal slices formed the basis of our investigation and it was not thought to be invalidated by the exclusion of small plaques occurring between cuts and the appearance of large plaques in more than one slice.

The cut coronal slices were photographed from the posterior aspect. On life size prints each plaque was outlined in ink, and its position recorded (Fig. 1). Every plaque was then represented by a dot on one of a series of photographs of normal coronal brain slices (Figs. 2 to 15). The dot was placed in the position of the apparent centre of the plaque, and in this way every plaque from all the cases was assigned to an anatomical situation. The amassed dots were then counted and assigned to various topographical categories, the first division being into left and right hemispheres. Four broad divisions were then made, namely, cortex, white matter, central grey matter, and the junction of cortex with white matter. Plaques were assigned to the last category only when they clearly appeared to be astride the boundary. These categories were further divided first into lobes and subsequently into named gyri.

\section{RESULTS}

The total number of plaques counted was 1,594 , making the average for each case 72 . Their relative distribution is recorded in Tables I and II. The percentages for the right and left hemispheres were 51 and 49 , and this equality appeared not only in the totals but also in every anatomical structure considered.

In order to compare the incidence of plaques in the various lobes of the cerebrum the area occupied

TABLE I

DISTRIBUTION OF 1,594 CEREBRAL PLAQUES IN 22 CASES OF MULTIPLE SCLEROSIS

\begin{tabular}{lc} 
Anatomical Localization & Number of Plaques \\
\hline Frontal lobe & $348(22 \%)$ \\
Parietal lobe & $233(15 \%)$ \\
Temporal lobe & $193(12 \%)$ \\
Occipital lobe & $14(1 \%)$ \\
Insula & $24(1 \%)$ \\
Corpus callosum & $60(4 \%)$ \\
Internal capsule & $19(1 \%)$ \\
Lateral ventricular system (periventricular) & $637(40 \%)$ \\
Internal nuclei & $61(4 \%)$ \\
Miscellaneous & \\
(Hypothalamus, red nucleus, subthalamic nucleus, & $(\mathrm{Less}$ than \\
amygdaloid nucleus, habenulopeduncular tract) & $5(1 \%)$
\end{tabular}

TABLE II

DISTRIBUTION OF 1,594 CEREBRAL PLAQUES IN 22 CASES OF MULTIPLE SCLEROSIS

Position in Grey or White Matter

Number of Plaques

Cortex $80 \quad(5 \%)$

Central grey matter

Junction of cortex and white matter

White matter

$265(17 \%)$

$1,184(74 \%)$ 


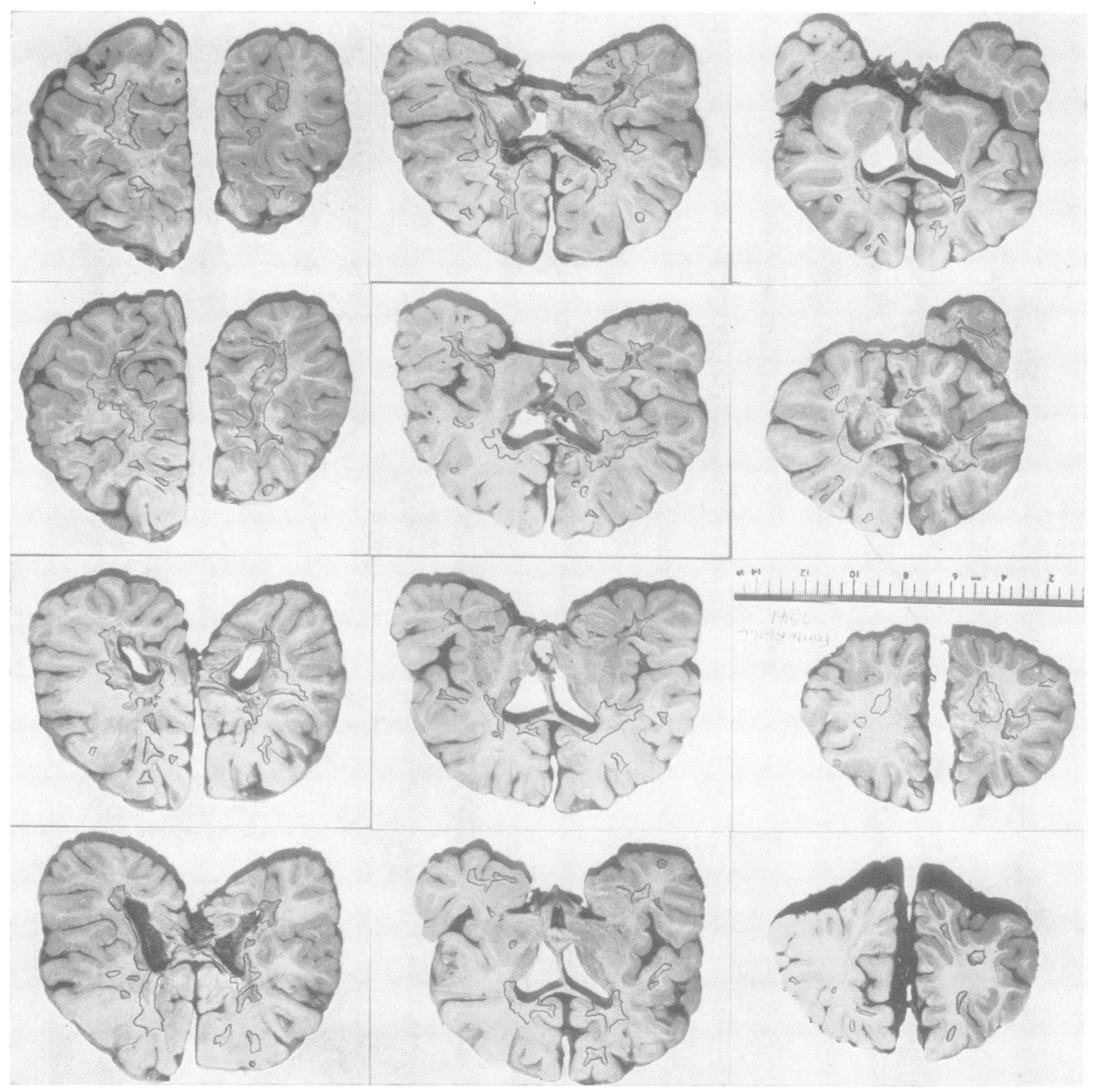

FIG. 1. Example of one case showing plaques outlined in ink on the coronal slices. 

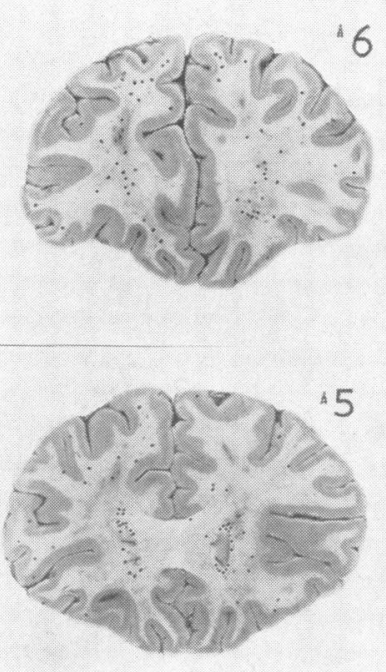

FIG. 2 (above) FIG. 3 (below)
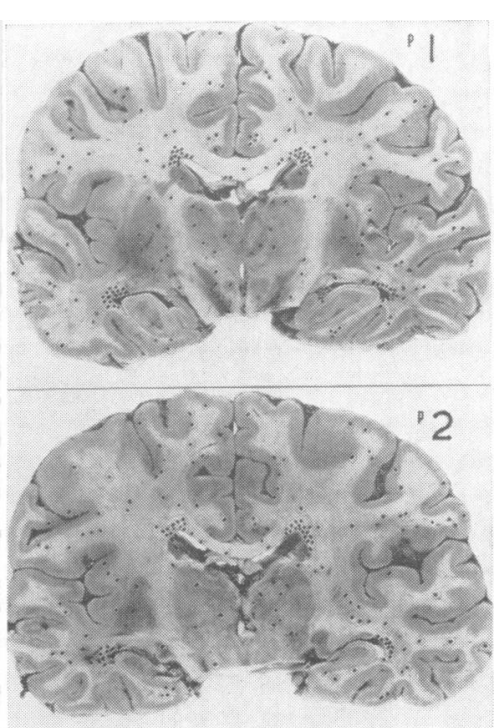

FIG. 8 (above) FIG. 9 (below)

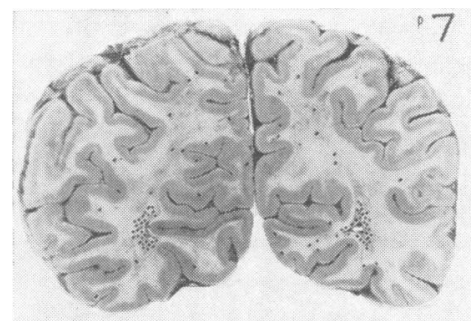

FIG. 14
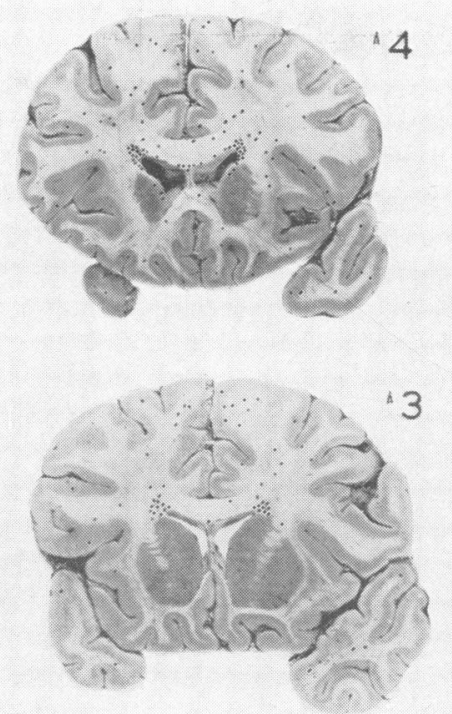

FIG. 4 (above) FIG. 5 (below)
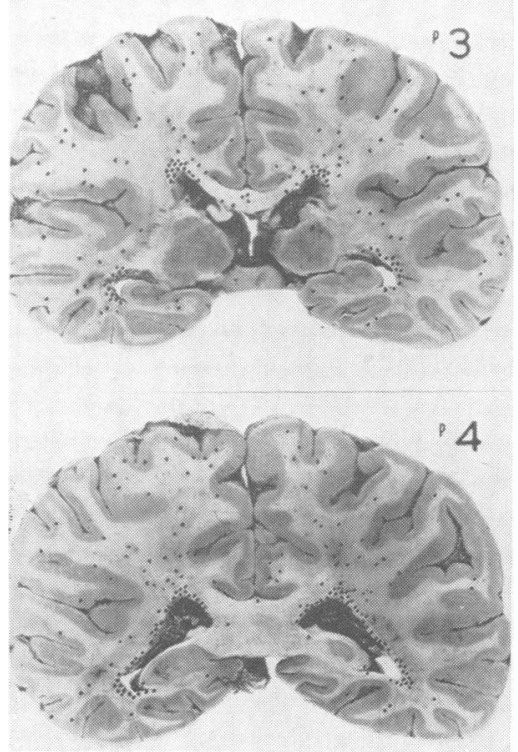

FIG. 10 (above) FIG. 11 (below)

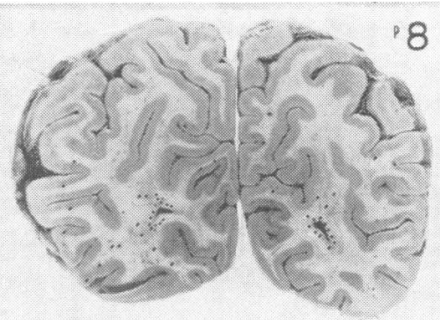

FIGS. 2 to 15. Photographs of coronal slices of normal cerebrum with dots representing the plaques seen in 22 cases of multiple sclerosis.
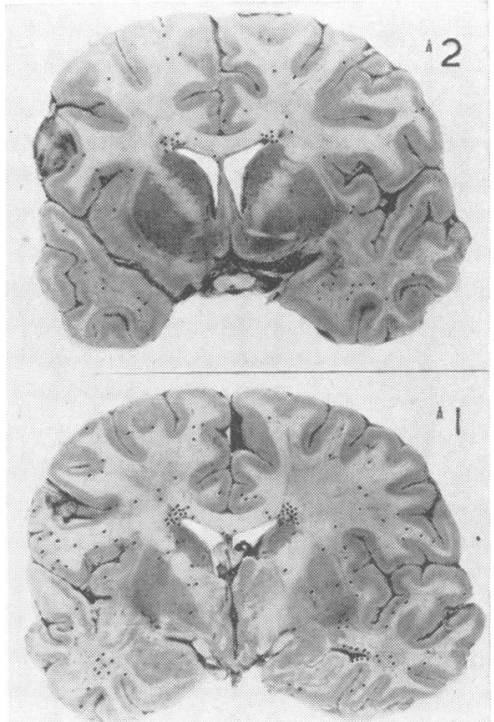

FIG. 6 (above) FIG. 7 (below)
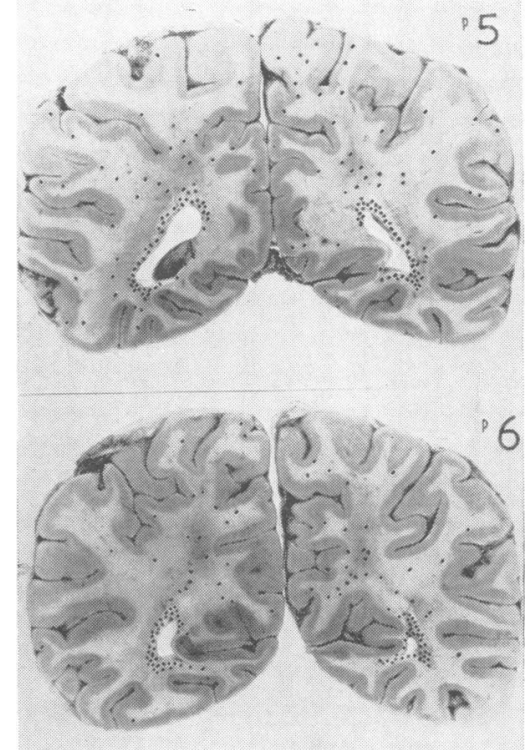

FIG. 12 (above) FIG. 13 (below) 

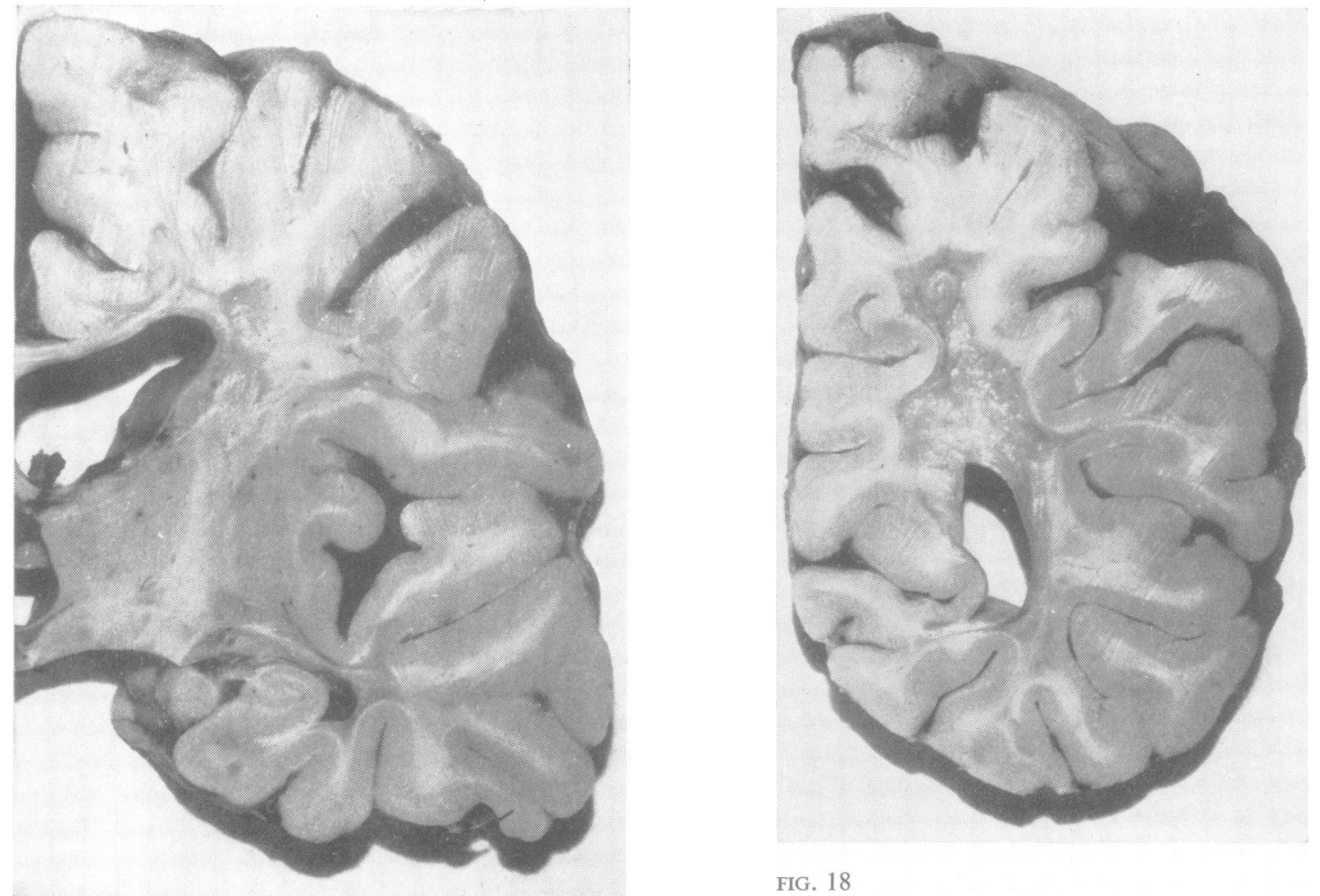

FIG. 16

FIGS. 16 and 18. Coronal slices of one of the cases studied. Note in Fig. 16 the periventricular plaques at the lateral angle of the lateral ventricle and around the inferior horn. In Fig. 18 extensive confluent plaques surround most of the posterior horn. In both pictures there are many plaques at the junction of cortex and white matter.

by each lobe on a series of standard coronal slices was measured by a planimeter. When the resulting figures were taken into consideration, it was found that the incidence of plaques per square centimetre in the various lobes was not significantly different.

Plaques in white matter formed $74 \%$ of the total, with $17 \%$ at the junction of cortex and white matter and $9 \%$ in the grey matter. When lesions in white matter were further divided into those occurring in periventricular regions and in remaining white matter, it was found that the former had the largest number, amounting to $40 \%$ of the total.

The number of plaques in each case varied enormously, the largest being 225 , and the smallest three. Compared with most cases material from three contained such large numbers of plaques $(225,220$, and 180$)$ as to suggest that the demyelinating process had been particularly active. In cases with very few plaques, these were almost always situated in relation to the posterior horns of the lateral ventricles. In general, large plaques were always periventricular, the largest being situated around the posterior (Fig. 18) and anterior horns (Fig. 16). Dilatation of the lateral ventricles was common, being moderately severe in 11 and very severe in three of the 22 cases. The ventricular dilatation appeared to be compensatory to loss of white matter and its degree was related to the number and size of the plaques.

Cortical plaques were difficult to see macroscopically and it is possible that histological examination might have shown more in this situation. The cortical plaques (total 80 in the 22 cases) were almost all from a single case, being one of the three with very numerous plaques.

\section{DISCUSSION}

The first point which emerges from this study is that all parts of the cerebrum are in some degree vulnerable to plaque formation. There was no difference between left and right hemispheres, and the varying 

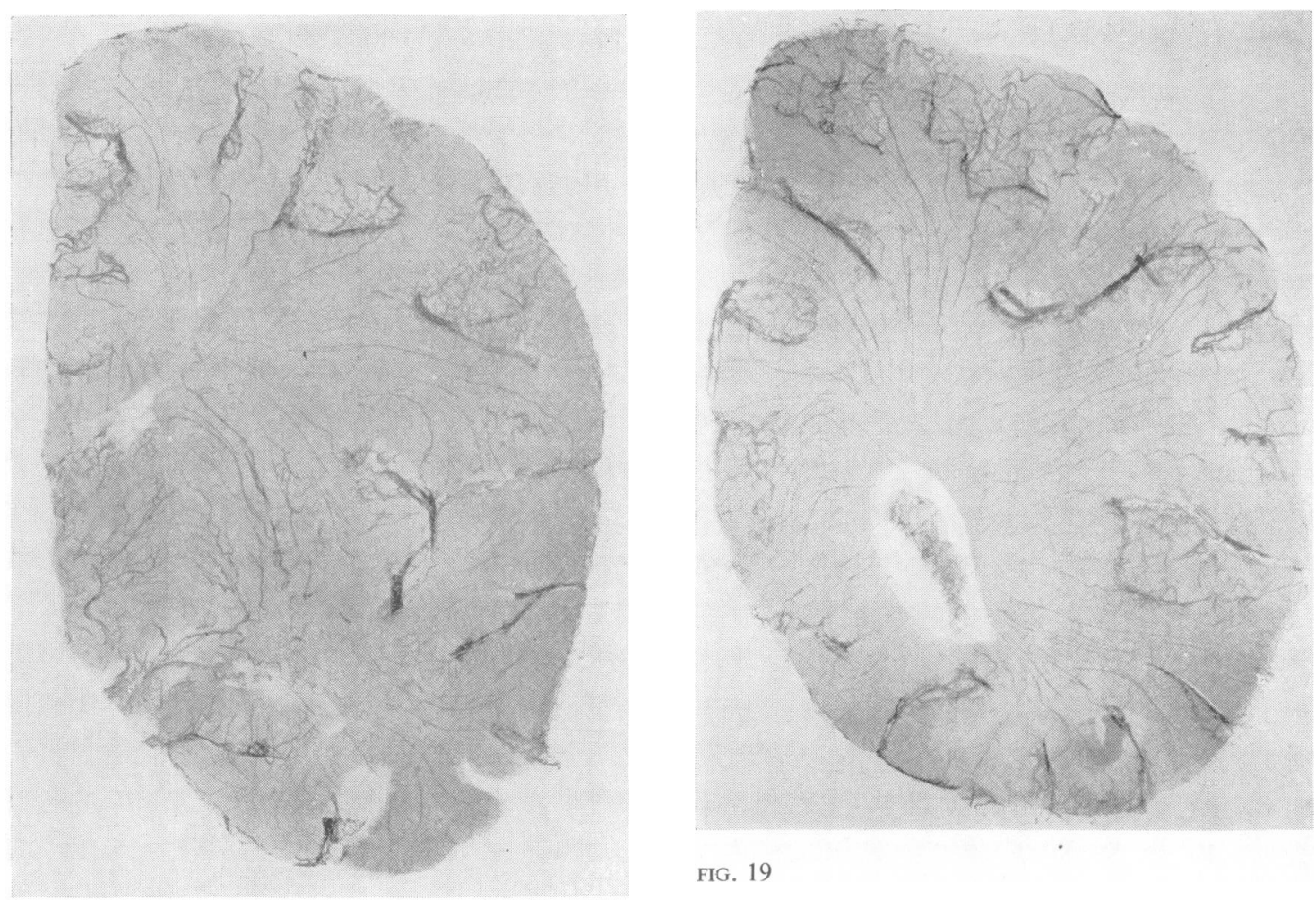

FIG. 17

FIGS. 17 and 19. Reproductions from Lazorthes (1961), showing injected coronal slices of normal brain. Note the avascularity of certain periventricular regions.

figures for individual lobes seemed related to their size. The plaques in the white matter were the more conspicuous but plaques were common at the junction of cortex and white matter and in the internal nuclei. This universal susceptibility must be borne in mind when theorizing about the anatomical sites where plaques are most common.

Two anatomical sites of predilection, however, merit separate consideration, namely, the periventricular and the junction of cortex and white matter. The term 'periventricular', although useful, does not state precisely where plaques commonly occur. Only the lateral ventricles show this susceptibility, for the third ventricle bears mural plaques no more commonly than elsewhere. There is a further striking localization to the supero-lateral angles of the anterior horns and of the bodies of the lateral ventricles. Plaques are also very common around the inferior and posterior horns and in these situations the plaques surround the horns apart from the infero-medial angles which are almost entirely spared. Equally important is the observation that certain large periventricular areas are not particularly susceptible. These include grey matter, such as the caudate nucleus and the thalamus, and white matter, chiefly the corpus callosum which has few plaques. It follows that theories involving a toxin circulating in the cerobrospinal fluid do not wholly explain the position of periventricular plaques. Such theories would require an additional explanation for the postulated toxin affecting these particular periventricular regions.

The idea of a vascular basis underlying multiple sclerosis has recurred constantly in the world literature and appears in the first full pathological description of the disease by Rindfleisch (1863) but the careful studies of Dow and Berglund (1942) and of Zimmerman and Netsky (1950) have refuted any direct causative connexion between plaques and diseased or thrombosed vessels. These conclusions have not discounted some more subtle peculiarity of the circulation in certain cerebral areas which may be 
important in determining the site of plaques. The periventricular regions we are considering have the pecularity that they are situated on the boundary zones between major cerebral arteries which have penetrated in this periventricular region to their further point of supply. That these areas are relatively avascular can be seen from the injection studies of Lazorthes (1961), two of whose illustrations from an extensive injection study of the major cerebral arteries appear in Figs. 17 and 19. In Fig. 17 it can be seen that the white matter at the lateral angle of the lateral ventricle contains few blood vessels. Furthermore these are derived from two independent major arteries (anterior and middle cerebral) which have here reached their furthermost point of supply. In contrast, those regions which, although adjacent to lateral ventricles, do not seem unduly susceptible to plaque formation, e.g., the thalamus, caudate nucleus, and hippocampus, appear to have an abundant arterial supply. In Fig. 19 there is a large, relatively avascular area extending around the lateral aspect of the posterior horn. This area is supplied by the terminal branches of long penetrating arteries which again arise from independent major arteries (anterior, middle, and posterior cerebral). These two areas (Figs. 17 and 19) are the regions where the greatest concentration of plaques was found in our study.

Less conspicuous than the periventricular situation but still striking was the common occurrence of plaques at the junction between cortex and white matter. It can be seen from Figs. 17 and 19 that the cortex has a rich blood supply from short cortical branches. The blood supply to the white matter depends on long penetrating arteries and it is possible that the junction between cortex and white matter is relatively avascular, and in this respect this area may be similar to the particular periventricular regions we have just considered.
SUMMARY

From 22 cases of multiple sclerosis the plaques as seen in $1 \mathrm{~cm}$. coronal slices were counted and their position recorded.

Plaques were commonest in relation to the lateral ventricles, the regions affected being the lateral angles of the anterior horns and bodies, and around the inferior and posterior horns. They were strikingly common astride the junction of cortex and white matter.

No significant difference in frequency was found between the left and right hemispheres, nor for individual lobes when the size of these was taken into consideration.

No region appeared immune from the occurrence of plaques.

It may be of aetiological significance that the sites where plaques were commonly formed are areas where relative vascular insufficiency can be postulated.

It is a pleasure to record our indebtedness to Dr. W. Ritchie Russell for his active encouragement at all stages of this work, to Mr. E. L. Tugwell for his advice and assistance with the photographs, and to Messrs. Masson et Cie for permission to reproduce Figs. 17 and 19. Dr. Betty Brownell is in receipt of a research grant from the Nuffield Foundation.

\section{REFERENCES}

Dow, R. S., and Berglund, G. (1942). Arch. Neurol. Psychiat. (Chic.), 47, 1 .

Fog, T. (1950). Ibid., 63, 382

Lazorthes, G. (1961). Vascularisation et Circulation Cérébrales. Masson, Paris.

Lumsden, C. E. (1955). In Multiple Sclerosis by McAlpine, D. Compston, N. D., and Lumsden, C. E. Livingstone, Edinburgh and London.

Rindfleisch, E. (1863). Virchows Arch. path. Anat., 26, 474.

Zimmerman, H. M., and Netsky, M. G. (1950). Res. Publ. Ass. nerv. ment. Dis., 28, 271. 